\title{
Early sleep triggers memory for early visual discrimination skills
}

\author{
Steffen Gais, Werner Plihal, Ullrich Wagner and Jan Born
}

Clinical Neuroendocrinology, Medical University of Lübeck, Ratzeburger Allee 160/Hs 23a, 23538, Lübeck, Germany

Correspondence should be addressed to J.B. (born@kfg.mu-luebeck.de)

\begin{abstract}
Improvement after practicing visual texture discrimination does not occur until several hours after practice has ended. We show that this improvement strongly depends on sleep. To specify the process responsible for sleep-related improvement, we compared the effects of 'early' and 'late' sleep, dominated respectively by slow-wave and rapid eye movement (REM) sleep. Discrimination skills significantly improved over early sleep, improved even more over a whole night's sleep, but did not improve after late sleep alone. These findings suggest that procedural memory formation is prompted by slow-wave sleep-related processes. Late REM sleep may promote memory formation at a second stage, only after periods of early sleep have occurred.
\end{abstract}

The consolidation of memories, a concept introduced a century ago $^{1}$, is particularly supported by processes occurring during sleep. The earliest studies on this topic ${ }^{2,3}$ and later animal and human studies ${ }^{4-6}$ provide considerable evidence that sleep helps to consolidate memories. Spatiotemporal patterns of neuronal activity are replayed in the rat hippocampus during periods of slow-wave sleep (SWS) following learning. This replay has been linked to consolidation of declarative types of (spatial) memory occurring during this sleep stage ${ }^{7,8}$. Consistent with these findings, human memory for word pairs and spatial locations benefits significantly more from early sleep dominated by extended SWS than from late sleep where REM sleep prevails ${ }^{9-11}$. Whereas these observations pertain to a declarative type of memory that relies mainly on the integrity of the hippocampus and adjacent temporal lobe structures, attention has only recently been focused on non-declarative, procedural types of memory ${ }^{10-13}$. Procedural knowledge refers to implicit (or pre-attentive) learning of 'habits' or 'how to' memories by practicing sensory and motor skills, respectively ${ }^{14}$. Unlike the declarative memory system, procedural memory does not necessarily involve hippocampal functions but, depending on the type of task, relies on various neocortical and subcortical structures ${ }^{15,16}$.

The performance improvement for a basic texture discrimination task takes place in assemblies of neurons active at a very early pre-attentive stage of visual processing ${ }^{17}$. Substantial improvement in perceptual performance of this task occurs eight or more hours after it has ended, rather than during or immediately after practice; this finding indicates a slow, latent process of learning ${ }^{18}$. Sleeping during the retention interval seems to be particularly important for improvement ${ }^{19}$. Selective disruption of REM sleep blocks overnight enhancement of the perceptual skill, whereas SWS disruption has no effect on enhancement ${ }^{12}$. These results led to the conclusion that the consolidation of texture discrimination, that is, enhancing the neural pathways involved in this task and thereby improving task performance, is a process strongly dependent on REM sleep.

However, the REM sleep deprivation protocol has been critiqued $^{20,21}$ because it induces distinct emotional and cognitive disturbances that interfere with task performance at the time of retrieval testing, thus making it difficult to make straightforward conclusions about the consolidation process ${ }^{22,23}$. To avoid these problems, we dissociated functions of REM sleep and SWS for visual procedural memory by splitting the night into two halves? In humans, the first half of sleep is normally dominated by periods of SWS, and there is little REM sleep. During late sleep, this pattern reverses. If REM sleep is essential for consolidation of discrimination skills, consolidation should be strengthened from a retention period encompassing predominantly REM sleep, rather than SWS, and vice versa. Accordingly, learning of texture discrimination skills was compared after nocturnal retention periods of either early or late sleep. In control conditions, subjects remained awake throughout corresponding retention periods. Improvement in texture discrimination was measured by comparing the minimum presentation time (stimulus to mask onset asynchrony, SOA) necessary to discriminate orientation of a target feature, before and after the retention period.

\section{RESULTS}

Sleep data confirmed that SWS dominated early sleep and REM sleep dominated late sleep (Table 1). During initial learning before sleep, texture discrimination performance did not differ between the early and late conditions ( $122 \pm 6.7 \mathrm{~ms}$ versus $120 \pm 6.3 \mathrm{~ms}, p>0.7)$. Subjects were retested after sleep, and their discrimination skills improved only after early sleep. During late sleep and during both early and late wake intervals, discrimination performance even decreased, that is, threshold SOA increased (Fig. 1a). This pattern was statistically confirmed by overall ANOVA with two within-group factors-threshold SOA before versus after the retention interval, and early versus late retention interval-and one between-group factor, sleep versus wake $\left(F_{1,25}=7.23, p<0.02\right.$ for before/after $\times$ sleep/wake interaction; $F_{1,25}=9.74, p<0.01$ for main effect of before/after). Separate analysis of the early and late conditions revealed this interaction to be significant for the early condition $\left(F_{1,13}=9.73\right.$, $p<0.01)$. Performance improvement, as measured by the decrease in threshold SOA, was significant after early sleep $\left(t_{7}=2.33, p<0.05\right)$, whereas wake controls showed an opposing tendency toward increasing thresholds across the early reten- 
tion interval $\left(t_{6}=-2.22, p<0.10\right)$. For the late condition, in which threshold SOA increased across the retention interval in both the sleep and wake groups, we found a significant main effect of before/after $\left(F_{1,12}=8.85\right.$, $p<0.02)$. Separate analysis of sleep and wake conditions confirmed a significant before/after $\times$ early/late interaction $\left(F_{1,13}=8.20, p<0.02\right)$ for sleeping subjects and a significant main effect of before/after $\left(F_{1,12}=10.39, p<0.01\right)$ for the wake control group, thus confirming a selective improvement in discrimination skills after the early retention sleep, and a decrease in performance especially after the wake periods.

In a supplementary study, we examined the improvement in texture discrimination after a 12-hour retention interval, which either was at night and contained an 8-hour period of sleep, or was during the day and did not contain a period of sleep. As expected from previous studies ${ }^{18}$, the threshold SOA in this experiment decreased from $130 \pm 14.3 \mathrm{~ms}$ before sleep to $105 \pm 12.1 \mathrm{~ms}$ after sleep $\left(t_{5}=6.07, p<0.001\right)$ in those subjects tested over a night retention interval. However, subjects' performance did not improve during the day retention interval of equal length $(141 \pm 10.9 \mathrm{~ms}$ before versus $143 \pm 13.4 \mathrm{~ms}$ after, $\left.t_{5}=-0.337, p>0.7\right)$. In addition, the improvement in discrimination skill during the full period of nocturnal sleep was compared with that observed in the main experiment during a period of early sleep alone. Whereas task performance before sleep was comparable for both of these conditions $(130 \pm 14.3 \mathrm{~ms}$ versus $122 \pm 6.7 \mathrm{~ms}, p>0.5$ ), the improvement during the entire night was about three times greater than the improvement during early sleep alone $\left(t_{12}=-3.23, p<0.01\right.$; Fig. $\left.1 \mathrm{~b}\right)$.

A second supplementary study focused on the possible influences of circadian rhythm on discrimination performance, and tested subjects on a well-learned task. As expected, discrimination task training led to an asymptotic reduction in threshold SOA (first session, $115 \pm 7 \mathrm{~ms}$; tenth session, $85 \pm 6 \mathrm{~ms}$ ). Perfor- mance on the well-learned task at 2200 hours, 0300 hours and 0800 hours was compared to performance on a task with a new target location, which differed at each test. The subjects' performance was comparable for all three times of testing, for familiar stimuli ( $82 \pm 14 \mathrm{~ms}, 79 \pm 10 \mathrm{~ms}$ and $86 \pm 17 \mathrm{~ms}$ respectively) and for novel stimuli (122 $\pm 26 \mathrm{~ms}, 120 \pm 23 \mathrm{~ms}$ and $134 \pm 23 \mathrm{~ms}$ ), excluding essential effects of circadian rhythm on discrimination skills ( $p>0.50$ for all comparisons). Performance on familiar stimuli was better than performance on novel stimuli $(p<0.001$ for all three tests).

\section{Discussion}

Data from the main study showed that texture discrimination skills during the night improved only if the retention interval contained SWS-dominated early sleep; during late sleep alone, threshold SOA even increased. Texture discrimination skills also deteriorated when subjects were kept awake during the retention phase (main effect across wake conditions). This pattern cannot be explained by circadian rhythms because discrimination skills improved when subjects slept during the early retention interval but did not improve when they were kept awake during this time. In addition, circadian rhythms did not influence performance on a well-learned or novel discrimination task. Because the wake control group was deprived of sleep, their fatigue at retrieval testing may have impaired texture discrimination performance. However, if the improvement in texture discrimination was only due to sleep before retrieval testing, improvement should also have occurred after late

Fig. 1. Improvements in visual discrimination skill. (a) Difference in threshold SO A between learning and retrieval testing, after retention intervals during the early or late half of the night (mean \pm s.e.m.). Subjects either slept for three hours during the retention interval, or were kept awake throughout the time interval. Asterisk, $p<0.05$, for difference between conditions. Threshold SOA decreased only across the early sleep retention condition. $\dagger, p<0.05$, for a comparison with learning performance before the retention interval. (b) Difference in threshold SO A between learning and retrieval testing after the early sleep retention interval (white bar), the 12-hour retention intervals of a whole night's sleep (black bar), and the awake daytime period (gray bar). Larger improvement in visual discrimination was observed after a whole night's sleep, than after a period of early sleep alone. ${ }^{* *} p<0.01$ for difference between conditions. $\dagger+p<0.01$ for a comparison with learning performance before the retention interval. (c) Individual performance curves for two subjects tested on the early sleep condition (circles) and late sleep condition (triangles). Filled symbols, performance at initial learning before sleep; open symbols, performance at retrieval testing after sleep. Horizontal line, $80 \%$ correct responses. Threshold SOA is the point where performance curves cross the horizontal line.
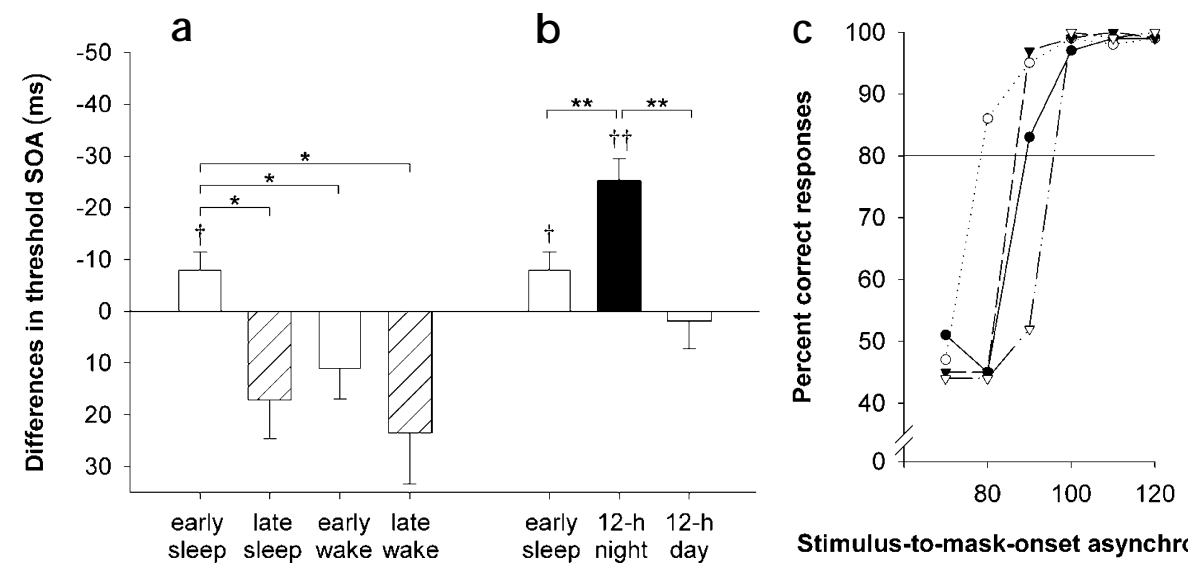
a

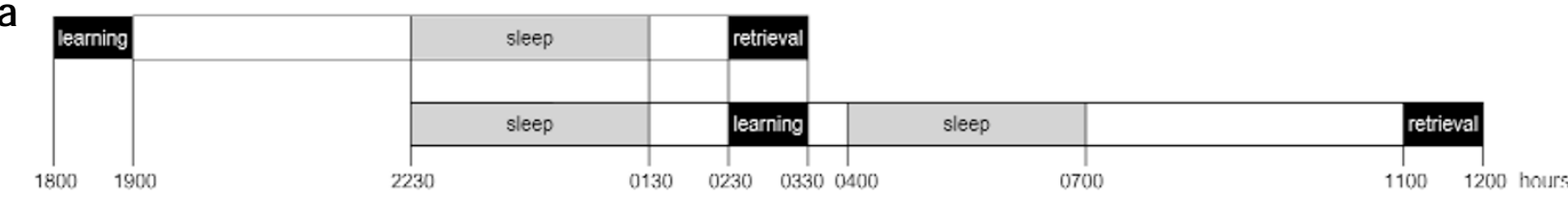

b

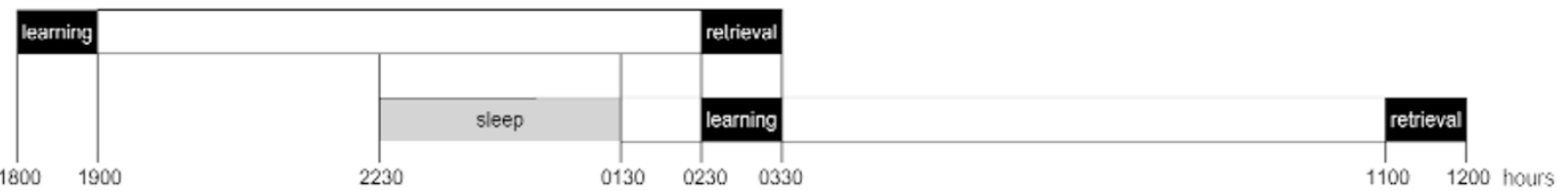

Fig. 2. Experimental protocol. Schedule (a) for the 'early' and 'late' sleep condition and (b) for the 'early' and 'late' wake control condition. Blank fields, times when subjects were awake; gray fields, intervals of sleep; black fields, time of texture discrimination task.

sleep. Moreover, initial task performance was very similar before early and late retention intervals, which argues against any considerable influence of fatigue or circadian variation.

The finding that performance improved only after early sleep suggests that processes related to this period of sleep facilitate consolidation of these procedural skills. The predominance of SWS and associated cortical changes in excitability ${ }^{24}$ and transmitter turnover ${ }^{25}$ may be an essential prerequisite for this facilitation. Besides SWS dominance, early sleep is also characterized by various neurohormonal changes, such as an inhibition of pituitaryadrenal release, which may be involved in memory consolidation. Although studies in rodents indicate memory enhancement for emotionally aversive tasks after administration of glucocorticoids (particularly into the basolateral amygdala ${ }^{26}$ ), human studies with systemic administration of glucocorticoids during early nocturnal sleep consistently show an impairment of declarative memory function during this period ${ }^{27,28}$.

The present findings contrast with results from a previous experiment, in which selective deprivation of REM sleep prevented an improvement in texture discrimination skill across nocturnal sleep, and deprivation of SWS sleep had no effect ${ }^{12}$.
Comparable amounts of time were spent in REM sleep during the REM sleep deprivation condition of that study ( $19 \pm 6 \mathrm{~min}$ ) and the early sleep condition of our study $(24.3 \pm 4.1 \mathrm{~min})$. The amounts of time spent in SWS during the SWS deprivation component of the previous study $(30 \pm 12 \mathrm{~min})$ and during the late sleep condition of our study $(31.6 \pm 4.1 \mathrm{~min})$ were also comparable. However, the REM sleep deprivation protocol of the previous study differed from our approach, in that this stage was disrupted after the REM sleep process was initiated. REM sleep deprivation leads to substantial fragmentation of sleep architecture. Resulting from the frequent arousals during REM sleep, emotional as well as attentive disturbances can be observed ${ }^{6,22,23}$. These disturbances particularly affect retrieval testing performance after sleep ${ }^{20,21}$. Considering our finding of improved texture discrimination after early sleep, it is difficult to determine why SWS deprivation did not disturb consolidation ${ }^{12}$. However, in the previous experiments, despite repetitive arousals, subjects spent an average of 30 minutes in SWS, in which the consolidation process may have been initiated ${ }^{29}$.

A supplementary experiment addressed two further issues. First, it indicated that sleep is necessary after practice to stimulate

Fig. 3. Task D isplays. Example of a stimulus (a) and a mask (b). All line positions varied slightly from trial to trial and the ' $T$ ' or ' $L$ ' in the center of the screen was rotated randomly. The target texture (three diagonal lines), upper left quadrant of the stimulus display (a).

\section{a}

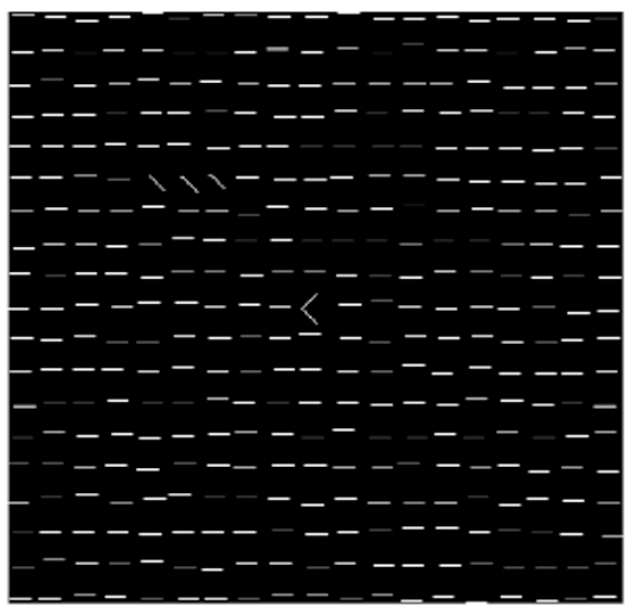

b

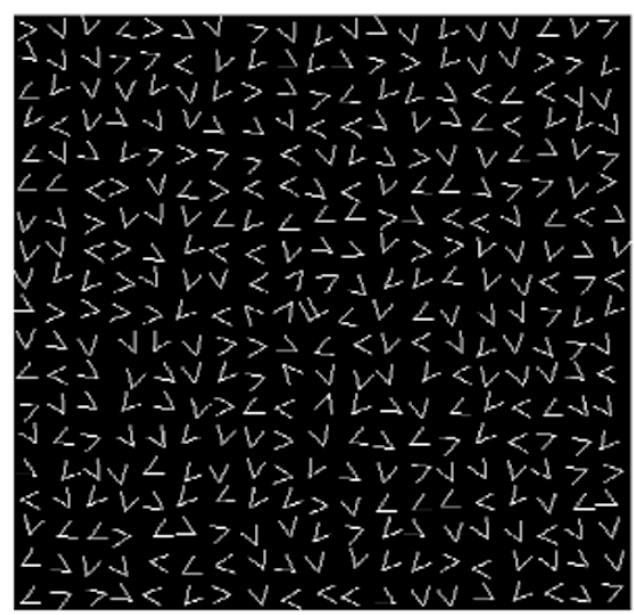


any consistent improvement in visual discrimination. When subjects were tested during the daytime wake phase, some subjects improved, whereas others deteriorated in performance. The failure of wake retention intervals to stimulate significant improvement in discrimination skills confirms previous data ${ }^{18,19}$. Second, when improvement after a full night of sleep was compared with improvement observed in the main experiment following early sleep alone, a striking interdependence of early and late sleep was revealed. The improvement across the entire sleep period was about three times greater than the improvement after early sleep alone (Fig. 1a). This difference was not only attributable to the longer retention interval across the full night, because a retention interval of equal length without sleep had no effect on task performance. Rather, the better performance after a full night of sleep suggests a two-step consolidation process.

Thus, although our data do not suggest that REM sleep is of primary importance for the learning of visual discrimination skills, REM sleep may add to consolidating memories once the effects of early sleep have been manifested. A previous correlational study ${ }^{19}$ used the same discrimination task as our study, and showed that the improvement in texture discrimination skills is correlated with the amount of time spent in SWS in the beginning of the night, and the time spent in REM sleep toward the end of the night. The present data extends this finding, and provides the first experimental evidence for a two-step process of memory formation during sleep, in which the second, REM sleep-related step is only effective after memory processes have been initiated in a first SWS-related step. Our experimental observations show that REM-rich late sleep alone is ineffective for memory consolidation, and that visual discrimination skills after an eight-hour sleep period (containing normal amounts of both SWS and REM sleep) are, on average, more than three times more improved than after a period of early sleep alone. The view of a two-step memory consolidation process would also integrate findings that arousals during REM sleep deteriorate consolidation of texture discrimination skills after early sleep and SWS have initiated consolidation ${ }^{12}$. Once memory traces have gained some strength, neocortical stimulation during REM sleep could lead to a reactivation of previously encoded materials, sharpening the traces ${ }^{30,31}$. Whether the small amount of REM sleep during early sleep (as well as the small amount of SWS in late sleep) have a particular function in this kind of sequential consolidation process remains to be determined.

Based on its local (retinotopic) nature, texture discrimination is considered to take place early during visual processing in the primary visual cortex and closely associated areas ${ }^{17,32}$. The learning of texture discrimination occurs at a pre-attentive level, and hence represents a fundamental type of procedural memory. In this regard, the selective improvement in discrimination skills after a period of early sleep diverges from previous studies in which a greater enhancement of procedural memory was observed after periods of late rather than early retention sleep ${ }^{10,11,13}$. One of those studies used a mirror-tracing task; the other used a word-stem priming task. Those tasks seem to be more complex than the texture discrimination task. The influence of REM sleep may become increasingly important with more complex tasks ${ }^{13,33,34}$, although the neurophysiological meaning of 'task complexity' in this context remains to be specified.

The neurophysiological mechanisms underlying sleep-associated facilitation of procedural memories are unclear. Improvement in texture discrimination skills may be a result of use-dependent changes in cell connectivity within V1 and closely connected areas ${ }^{17,32}$. These changes might involve processes of synaptic long-term potentiation and activity-dependent synaptogenesis known to depend on modulatory influences from certain neurotransmitters and neurohormonal inputs ${ }^{35-39}$. Experimental variation of transmitter and neurohormonal levels during selected sleep periods is a possible approach to determining the synaptic mechanisms involved in the different steps of consolidation.

\section{Metrods}

Subjects were healthy non-smokers (19-35 years old), with normal or corrected to normal vision. They slept seven to nine hours per night, and had no major disruptions of the sleep-wake cycle during the six weeks before experimentation. They were not allowed to ingest caffeine or alcohol, or sleep during the day before experimental nights. Before the experiment, subjects were accustomed to sleeping under laboratory conditions. The experiments were approved by the Ethics Committee of the Medical University of Lübeck.

In the main experiment, each of 15 subjects participated in two experimental nights, which were about one week apart. On these nights, subjects learned a texture discrimination task with the retention interval between learning and retrieval testing encompassing either the early or late half of the night. Subjects were randomly assigned to either a sleep group $(n=8)$, which had a 3 -hour period of sleep during the retention interval, or a control group, which remained awake during the retention interval $(n=7)$.

All sleep and wake periods could vary to a limited degree, to individually adapt them to a subject's normal sleep-wake rhythm (Fig. 2), but eight hours between learning and retrieval testing were required, because otherwise, no improvement could be expected for the task ${ }^{18}$. Sleep time was measured from sleep onset. Subjects were woken during the first stage-two sleep occurring after three hours of sleep. During the time the subjects were awake, they were not allowed to visually or physically strain themselves. Most of the time, they played board games or listened to music.

Two supplementary experiments were done to control for effects of circadian rhythm on retention and discrimination performance, respectively. In the first experiment, 12-hour retention intervals between learning and retrieval testing encompassed either nighttime ( 2100 hours-0900 hours; $n=6$ ) or daytime ( 0900 hours- 2100 hours; $n=6$ ). The nighttime interval included a complete 8-hour sleep interval beginning at 2300 hours. In the second supplementary experiment, seven subjects were tested on a well-learned task. They reported to the laboratory for 10 consecutive days, practicing the discrimination task with the target displayed in one location. Afterward, subjects spent one experimental night in the laboratory, being tested for discrimination performance at 2200 hours, 0300 hours and 0800 hours. They slept two times for three hours during these nights (2300-0200 hours and 0400-0700 hours). At each test, performance on the well-learned task was compared to performance on a novel discrimination task, with the target displayed in a new location.

The visual discrimination task was designed as described ${ }^{17}$. It was carried out in a silent and dark environment. Stimuli were presented on a Macintosh PowerPC computer with a 17 -inch monitor $(75 \mathrm{~Hz})$. Subjects were asked to react by pressing keys on a keyboard. Each session consisted of 1250 trials, each composed of three sequential displays. First, a cross was displayed in the center of the screen. Subjects were told to leave their eyes fixed at this point throughout the trial. After they pressed a key, there was a blank screen interval of 250-300 ms. Second, the target display (Fig. 3a) was shown for $10 \mathrm{~ms}$, followed by another blank screen interval. Third, the mask was presented for $100 \mathrm{~ms}$ (Fig. 3b). Exposure to the mask overrode the remains of the target display on the retina. Thus, discrimination difficulty could be systematically increased by reducing the stimulus to mask onset asynchrony (SOA).

The target displays were $16^{\circ}$ of visual angle in size and contained a field of $19 \times 19$ horizontal bars with a randomly rotated 'T' or 'L' shaped figure in the center. The target (three horizontally or vertically aligned diagonal bars) was located in the peripheral visual field at a distance of $3^{\circ}-5^{\circ}$ of visual angle from the center. After each trial, subjects had to report by key press the letter in the center of the display and the target orientation, that is, the alignment of the three diagonal lines. Discrimination of the cen- 
tral letter assured correct eye fixation, and only trials with correct letter discrimination were used for analysis. There was no time limit for giving the answer, and subjects were told to take breaks when needed. SOA was continuously reduced after a block of trials. Steps were 460, 360, 260 and $220 \mathrm{~ms}$ with 50 trials per step. Then SOA was reduced from 180 to $60 \mathrm{~ms}$ in steps of $20 \mathrm{~ms}$, with 150 trials at each step. Thus, a total of 1250 trials were done for each period of learning and retrieval testing. Before the experiment, subjects practiced the task in the presence of the experimenter with 50 trials at a SOA of $460 \mathrm{~ms}$. Performance was measured as the percent correct responses at a given SOA. Threshold SOA was estimated by interpolating the point where the recognition rate was $80 \%$. Thus, improvement in texture discrimination between learning and retrieval testing was equivalent to a reduction in threshold SOA. Sessions with a threshold SOA of more than $220 \mathrm{~ms}$ were excluded from analysis, because they were clearly recognizable as outliers.

The target was shown in the same quadrant of the visual field during learning and subsequent retrieval testing after a retention period, but different quadrants were used on the two experimental conditions of a subject. Because improvement in this task is retinally local, that is, it occurs only within the area of the visual field where the target is repeatedly presented ${ }^{17}$, improvement measured in two different quadrants of the visual field can be assumed to be independent. This was confirmed by supplementary analyses of our data.

\section{ACKNOWLEDGEMENTS}

We thank A. Karni and R. Stickgold for providing the software for the texture discrimination task, and for comments on the manuscript. This research was supported by a grant from the Deutsche Forschungsgemeinschaft (DFG) to J.B.

\section{ReCeIved 21 August; ACCePTed 20 SePtember 2000}

1. Müller, G. E. \& Pilzecker, A. Experimentelle beiträge zur lehre vom gedächtnis. Z. Psychol. 1, 1-300 (1900).

2. Heine, R. Uber wiedererkennen und ruckwirkende hemmung. Z. Psychol. 68, 161-236 (1914).

3. Jenkins, J. G. \& Dallenbach, K. M. Obliviscence during sleep and waking. Am. J. Psychol. 35, 605-612 (1924).

4. Hennevin, E., Hars, B., Maho, C. \& Bloch, V. Processing of learned information in paradoxical sleep: relevance for memory. Behav. Brain Res. 69, 125-135 (1995).

5. Smith, C. Sleep states and memory processes. Behav. Brain Res. 69, 137-145 (1995).

6. Cipolli, C. Sleep, dreams and memory: an overview. J. Sleep Res. 4, 2-9 (1995).

7. Wilson, M. A. \& McNaughton, B. L. Reactivation of hippocampal ensemble memories during sleep. Science 265, 676-679 (1994).

8. Buzsaki, G. Memory consolidation during sleep: a neurophysiological perspective. J. Sleep Res. 7, 17-23 (1998)

9. Yaroush, R., Sullivan, M. J. \& Ekstrand, B. R. Effect of sleep on memory. II. Differential effect of the first and second half of the night. J. Exp. Psychol. 88, 361-366 (1971)

10. Plihal, W. \& Born, J. Effects of early and late nocturnal sleep on declarative and procedural memory. J. Cogn. Neurosci. 9, 534-547 (1997).

11. Plihal, W. \& Born, J. Effects of early and late nocturnal sleep on priming and spatial memory. Psychophysiology 36, 571-582 (1999).

12. Karni, A., Tanne, D., Rubenstein, B. S., Askenasy, J. J. \& Sagi, D. Dependence on REM sleep of overnight improvement of a perceptual skill. Science 265 , 679-682 (1994).
13. Aubrey, J., Smith, C., Tweed, S. \& Nader, R. Cognitive and motor procedural tasks are dissociated in REM and stage two sleep. Sleep Res. Online 2, 220 (1999).

14. Squire, L. R. \& Zola, S. M. Structure and function of declarative and nondeclarative memory systems. Proc. Natl. Acad. Sci.USA 93, 13515-13522 (1996).

15. Pascual-Leone, A., Grafman, J. \& Hallett, M. Modulation of cortical motor output maps during development of implicit and explicit knowledge. Science 263, 1287-1289 (1994)

16. Pascual-Leone, A. et al. Procedural learning in Parkinson's disease and cerebellar degeneration. Ann. Neurol. 34, 594-602 (1993).

17. Karni, A. \& Sagi, D. Where practice makes perfect in texture discrimination: evidence for primary visual cortex plasticity. Proc. Natl. Acad. Sci.USA 88, 4966-4970 (1991).

18. Karni, A. \& Sagi, D. The time course of learning a visual skill. Nature 365, 250-252 (1993).

19. Stickgold, R., Whidbee, D., Schirmer, B., Patel, V. \& Hobson, J. A. Visual discrimination task improvement: A multi-step process occurring during sleep. J. Cogn. Neurosci. 12, 246-254 (2000).

20. Vertes, P. \& Eastman, K. The case against memory consolidation in REM sleep. Behav. Brain Sci. (in press).

21. Born, J. \& Gais, S. REM sleep deprivation: the wrong paradigm leading to wrong conclusions. Behav. Brain Sci. (in press).

22. Horne, J. A. \& McGrath, M. J. The consolidation hypothesis for REM sleep function: stress and other confounding factors-a review. Biol. Psychol. 18, 165-184 (1984).

23. Oniani, T. N. Does paradoxical sleep deprivation disturb memory trace consolidation? Physiol. Behav. 33, 687-692 (1984).

24. Marshall, L., Molle, M., Fehm, H. L. \& Born, J. Scalp recorded direct current brain potentials during human sleep. Eur. J. Neurosci. 10, 1167-1178 (1998).

25. McCormick, D. A. Neurotransmitter actions in the thalamus and cerebral cortex. J. Clin. Neurophysiol. 9, 212-223 (1992).

26. Roozendaal, B. Glucocorticoids and the regulation of memory consolidation. Psychoneuroendocrinology 25, 213-238 (2000).

27. Plihal, W., Pietrowsky, R. \& Born, J. Dexamethasone blocks sleep induced improvement of declarative memory. Psychoneuroendocrinology 24, 313-331 (1999).

28. Plihal, W. \& Born, J. Memory consolidation in human sleep depends on inhibition of glucocorticoid release. Neuroreport 10, 2741-2747 (1999).

29. Born, J., Muth, S. \& Fehm, H. L. The significance of sleep onset and slow wave sleep for nocturnal release of growth hormone $(\mathrm{GH})$ and cortisol. Psychoneuroendocrinology 13, 233-243 (1988).

30. Giuditta, A. et al. The sequential hypothesis of the function of sleep. Behav. Brain Res. 69, 157-166 (1995).

31. Maquet, P. et al. Reactivation during rapid eye movement (REM) sleep of cerebral areas involved in the execution of a serial reaction time (SRT) task. Sleep Res. Online 2, 694 (1999).

32. Schoups, A. A. \& Orban, G. A. Interocular transfer in perceptual learning of a pop-out discrimination task. Proc. Natl. Acad. Sci. USA 93, 7358-7362 (1996).

33. Empson, J. A. \& Clarke, P. R. Rapid eye movements and remembering. Nature 227, 287-288 (1970).

34. Smith, C. Sleep states, memory processes and synaptic plasticity. Behav. Brain Res. 78, 49-56 (1996).

35. Kirkwood, A., Rioult, M. C. \& Bear, M. F. Experience-dependent modification of synaptic plasticity in visual cortex. Nature 381, 526-528 (1996).

36. Kirkwood, A., Rozas, C., Kirkwood, J., Perez, F. \& Bear, M. F. Modulation of long-term synaptic depression in visual cortex by acetylcholine and norepinephrine. J. Neurosci. 19, 1599-1609 (1999).

37. Edagawa, Y., Saito, H. \& Abe, K. Serotonin inhibits the induction of longterm potentiation in rat primary visual cortex. Prog. Neuropsychopharmacol. Biol. Psychiatry 22, 983-997 (1998).

38. McEwen, B. S. Neurosteroids. (eds. Baulieu, E. E., Robel, P. \& Schumacher, M.) 233-254 (Humana, Totowa, New Jersey, 1999).

39. Cahill, L. \& McGaugh, J. L. Modulation of memory storage. Curr. Opin. Neurobiol. 6, 237-242 (1996). 